\title{
Mitigating the Effects of Interference in a Multipath Prone Environment for a DVB-T Transmission
}

\author{
Oluwaseun Y. Alabi and Michael O. Kolawole \\ Department of Electrical \& Electronics Engineering, School of Engineering \& Engineering Technology, \\ Federal University of Technology P.M.B 704, Akure, Ondo State, Nigeria
}

\begin{abstract}
This paper develops a novel simulation method that seeks to mitigate the effects of interference in a multipath prone environment for a DVB-T transmission using the AWGN and the Multipath Rayleigh Fading Channel. The channel parameters were optimized until desired result was obtained. The reception performance of the DVB-T system shows that interference though not totally eliminated, can be minimized while transmitting in a multipath prone environment.
\end{abstract}

Key Words: DVB-T, AWGN, Multipath Rayleigh Fading Channel

\section{Introduction}

The Digital Video Broadcasting -Terrestrial (DVB-T) is a transmission method developed by the DVB group with a first broadcast made in 1998 in the UK. The method offers transmission with a wider coverage, better picture quality and possibility for multiplexing of signals. DVB-T uses Orthogonal Frequency Division Multiplexing (OFDM) as its modulation technique. The OFDM is an established and widely accepted technique for high rate data communications due to its numerous advantage especially its high robustness against severe channel conditions. The DVB-T system uses basically 2048 or 8192 carriers (mode 2k or 8k) [ETSI, 2008]. Each of (data) carriers is modulated by the same digital quadrature modulation: currently and typically, QPSK (4PSK), 16QAM, and 64QAM. Two error correction techniques have been shown to decrease error bit rate in DVB-T system - the first is the Reed-Solomon technique and secondly the convolution coding with Viterbi decoding [Kolawole, 2002]. A code rate is chosen from a set of 1/2, 2/3,3/4, 5/6, and 7/8. Guard Interval GI-a cyclic prefix - is further inserted into the OFDM symbol to prevent influence of multipath transmission (i.e. the GI last, detection is stopped and delays could fade away) and facilitate easy synchronization and equalization [Karel, et.al, 2012]. The ratio between time of GI and time of useful part of symbol is chosen from a set of $1 / 4$, $1 / 8,1 / 16$, and $1 / 32$. The useful part of symbol length is $224 \mu$ s in mode $2 \mathrm{k}$ and $896 \mu$ s in mode $8 \mathrm{k}$.

\section{The Channels}

Two channels are considered in this paper: an additive noise-which is assumed to be stationary and independent Gaussian random variable (AWGN), and multipath Rayleigh fading - due to structural obstructions in the propagation path(s)associated with Doppler shift due to the receiver moving away from or towards the transmitter.

Since noise power does not appear just at one frequency but over a range of frequencies, noise power density $N_{o}$ is used, and the effect of noise in AGWN channel was observed as the noise level $N_{o}$ increases relative to carrier-signal strength $\mathrm{E}_{\mathrm{b}}$.

\section{Methodology}

The Model parameters as listed in Table1.0 were used in designing the DVB-T System. The major source of interference was the Multipath Rayleigh Fading Channel.

\begin{tabular}{|c|c|}
\hline Parameters & Modulation type: $2 \mathrm{k}$ 64-QAM \\
\hline Subcarriers & 2048 \\
\hline Puncture Vector & {$\left[\begin{array}{lllllll}1 & 1 & 0 & 1 & 1 & 0\end{array}\right]$} \\
\hline M-ary Number & 64 \\
\hline Number of OFDM Symbols & 6 \\
\hline QAM Symbols & 1512 \\
\hline Zeros Added & 536 \\
\hline Channel Model & AWGN \& Rayleigh Fading Channel \\
\hline
\end{tabular}

The system was designed to observe the effect of interference and to determine a possible value for which a minimum error is obtainable in a DVB-T transmission for fixed or mobile reception. The Model Fig. 1.0 shows the input data considered as any random integer that generates uniformly distributed random integers in the 
range [0, M-1] where $\mathrm{M}$ is the $\mathrm{M}$-ary modulation value, which equates to $\mathrm{M}=2^{\mathrm{b}}$ where $b$ is the bit-block [Kolawole, 2002]. Serial codes are generated by the encoder with the data sequence arranged and re-arranged by the outer and inner interleaver respectively to reduce the effect of burst errors while the first and second error level correction occurs at the outer and inner coder.

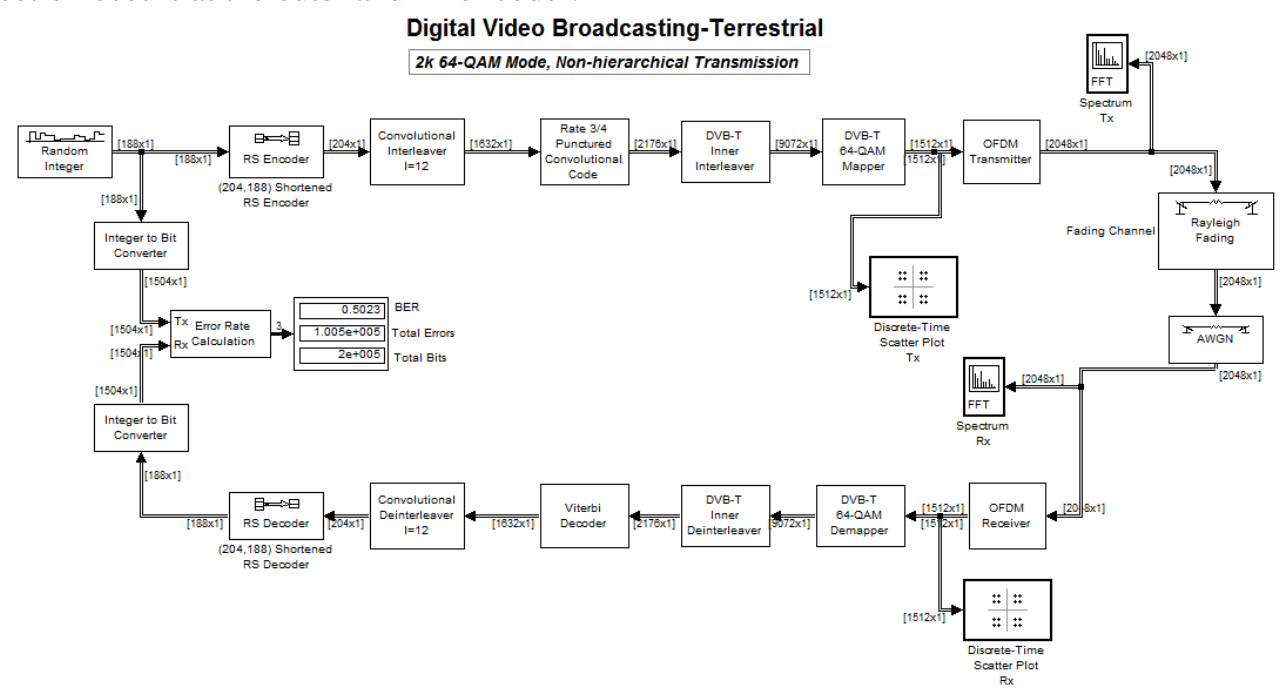

Figure 1.0 DVB-T Block Diagram Using SIMUINK

\section{Results \&Analysis}

The signal transmitted with no interference is seen in Fig. 1.1a and 1.1b using the spectrum scope and signal constellation mapping respectively.

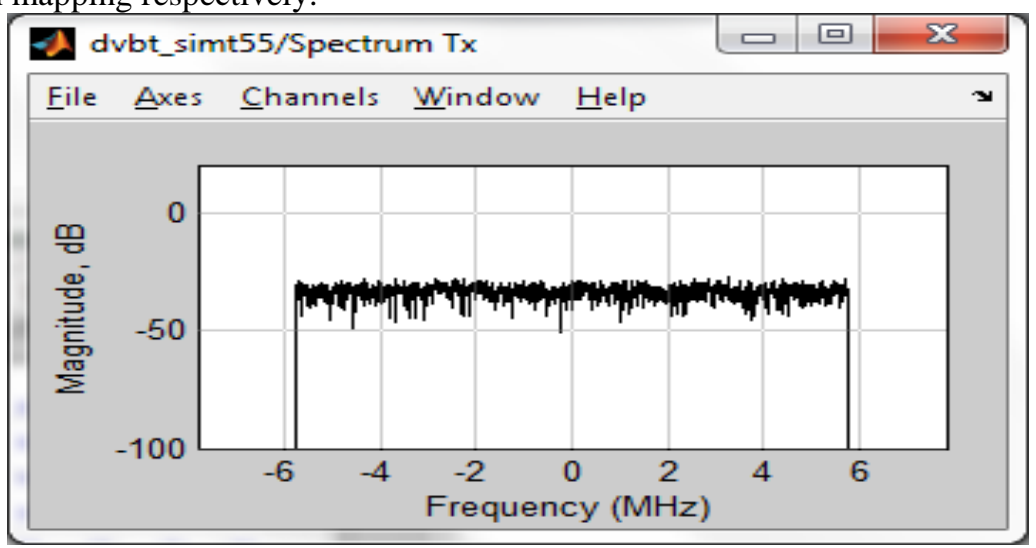

Figure 1.1(a) Spectrum Scope of the transmitted signal

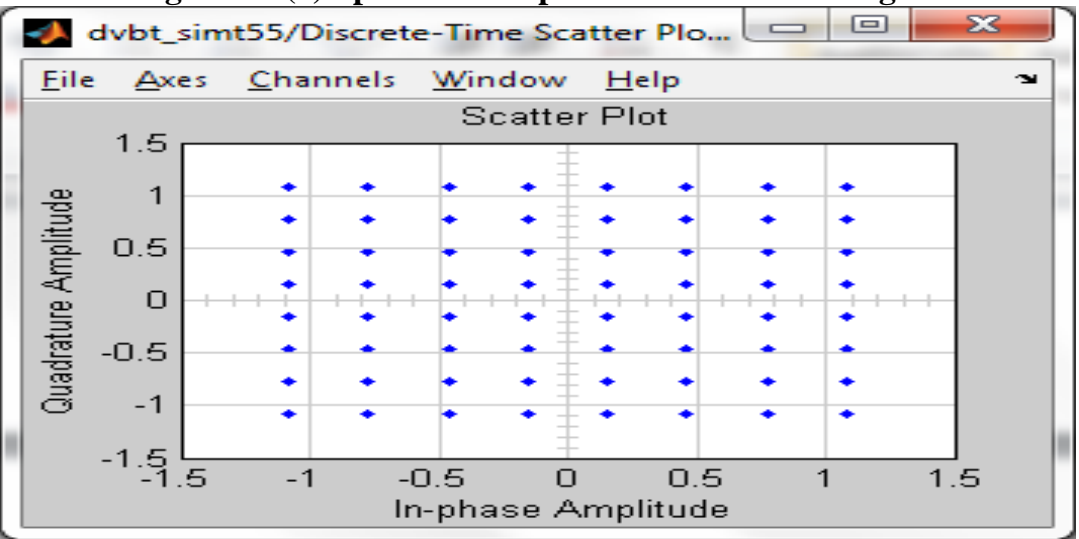

Figure 1.1(b) Signal Constellation of Transmitted signal

The transmitted signal under the influence of varying channel interferences was observed with the listed effects very visible (see Fig.1.2-1.3):

- Multiple distortion and Fading

- Loss of Orthogonality 
- Smearing of transmitted signal

- Loss of transmitted signal

- Signal/bits convergence

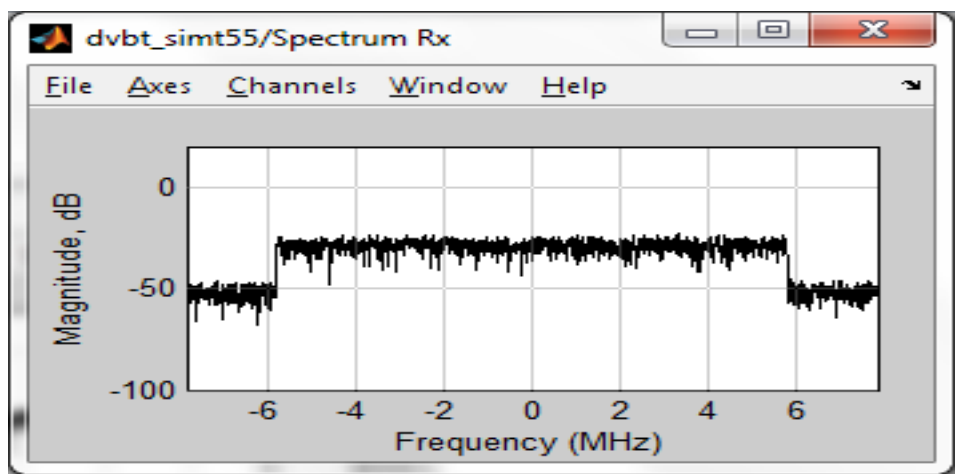

Figure 1.2 Spectrum Scope of the received signal at $5 \mathrm{~Hz}$

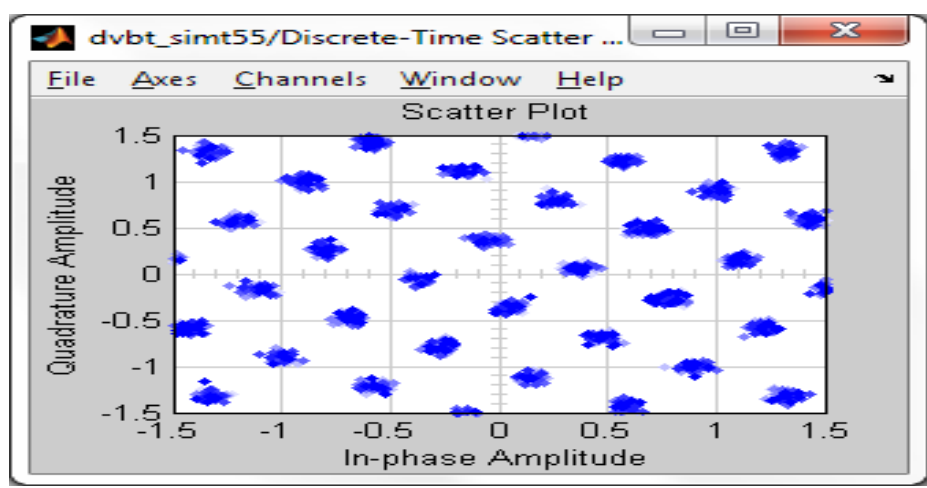

Figure 1.3 Signal Constellation received with less noise

To achieve minimum error transmission using both channels, the system parameter was optimized as follows:

1. $\mathrm{Eb} / \mathrm{No}$ for $\mathrm{AWGN} \quad-\quad 30 \mathrm{~dB}$

2. Doppler Shift for Rayleigh $\quad-\quad 50 \mathrm{~Hz}$

3. Delay Vector for Rayleigh - $\quad$ [0 1e-12]

Simulation result shows that lowest error, minimal BER and maximum number of bits transmitted, less fading and distortion was obtained with good signal constellation (Fig. 1.4). A further shift from these parameters shows a very poor reception as shown in Fig. 1.5.

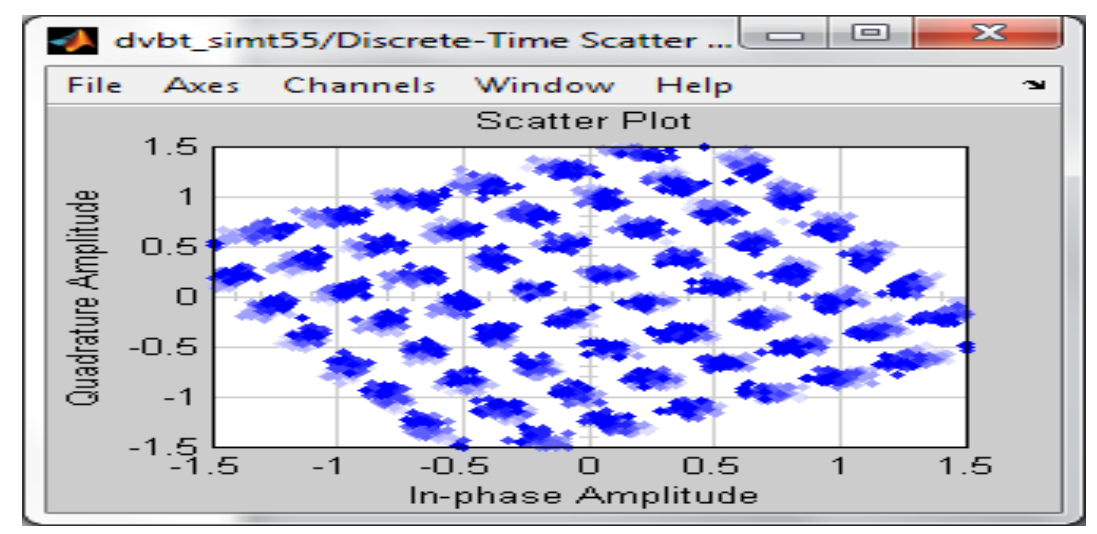

Figure 1.4 Signal Constellation received with fading at $50 \mathrm{~Hz}$. 


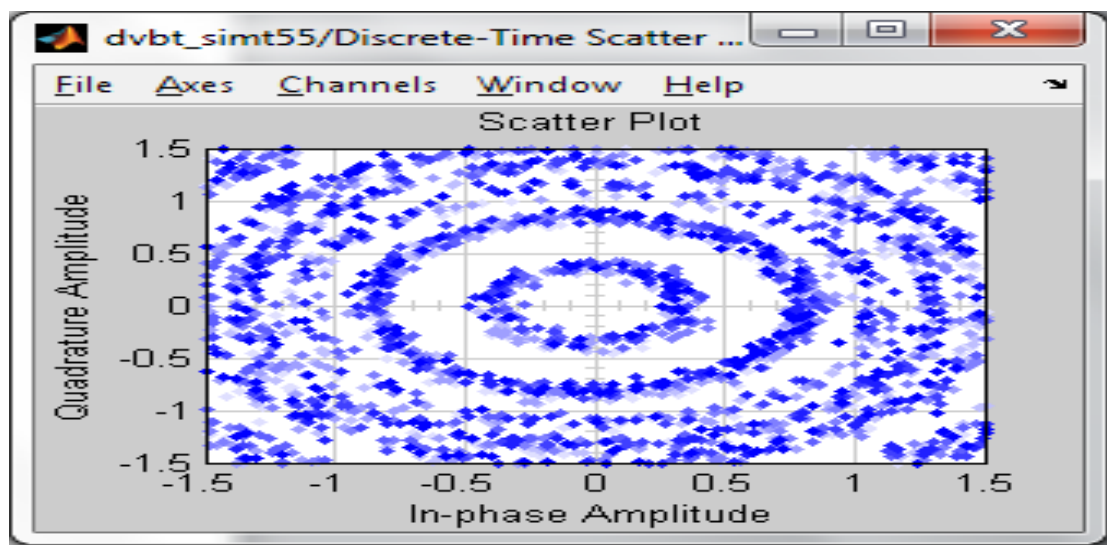

Figure 1.5 Signal Constellation using delay vector of [0 1e-9]

\section{Conclusion}

The combination of both noisy Channels was used in the establishment of a minimum error level condition for a DVB-T Transmission in a multipath prone environment. The reception performance of the DVB$\mathrm{T}$ system shows that interference though not totally eliminated can be minimized while transmitting in a multipath prone environment. The research work has shown that the $2 \mathrm{k}$ carrier modulation enhance much robustness against Doppler Frequency Shift.

The AWGN channel has also proven to be the best channel for DVB-T because it does not pose the challenge of multipath fading, distortion and loss of orthogonality of the system as seen in Rayleigh Multipath Fading Channel. This conclusion is consistent with Aamir et.al. [2012].

\section{References}

[1]. Aamir Khan, Syed Hassan Ahmed, Syed Muhammed Umar Talha, (2012) "Performance $\quad$ Evaluation of DVB-T Based OFDM over Wireless Communication Channels" Proceedings of IMECS.

[2]. ETSI (European Telecommunications Standards Institute) (2009), "Digital Video Broadcasting (DVB): Framing Structure, Channel Coding and Modulation for Digital Terrestrial Television" ETSI EN 300 744, vol. 1.6.1 (2009-01).

[3]. Karel Mikulastik, Karel Ulovec, (2012) "Multipath Propagation in Digital Video Broadcasting-Terrestrial System"

[4]. Kolawole, M.O. (2002). Satellite Communication Engineering, New York: Marcel Dekker 